\title{
The antidepressant effects of apigenin are associated with the promotion of autophagy via the mTOR/AMPK/ULK1 pathway
}

\author{
XIAOLONG ZHANG ${ }^{1-3}$, HONGMIN BU $^{3}$, YAN JIANG ${ }^{3}$, GUANGDA SUN $^{2}$, RUIZHI JIANG ${ }^{2}$, \\ XIAOYAN HUANG ${ }^{2}$, HUIFANG DUAN ${ }^{1,2}$, ZHIHENG HUANG ${ }^{1,2}$ and QINAN WU ${ }^{1,2}$ \\ ${ }^{1}$ College of Pharmacy, Nanjing University of Chinese Medicine; ${ }^{2}$ Jiangsu Collaborative \\ Innovation Center of Chinese Medicinal Resources Industrialization, Nanjing, Jiangsu 210023; \\ ${ }^{3}$ Lianyungang Food and Drug Inspection Center, Lianyungang, Jiangsu 222000, P.R. China
}

Received January 6, 2019; Accepted June 6, 2019

DOI: $10.3892 / \mathrm{mmr} .2019 .10491$

\begin{abstract}
The present study aimed to investigate whether apigenin elicits antidepressant effects in depressant-like mice via the regulation of autophagy. The depressant-like behaviors were established in a chronic restraint stress model. Male BALB/c mice were subjected to restraint stress for $6 \mathrm{~h} /$ day for a period of 21 days, and deficits in sucrose preference, tail suspension and forced swim tests were confirmed to be improved following oral apigenin. To investigate the underlining mechanisms, the hippocampal levels of p62 and microtubule-associated protein light chain 3-II/I (LC3-II/I) were measured using western blot analysis. The expression levels of LC3-II/I and p62 indicated that the significantly inhibited autophagy level induced by chronic restraint stress can be increased following apigenin treatment. Similar to the level of autophagy, the expression levels of adenosine monophosphate-activated protein kinase (AMPK) and Unc-51 like autophagy activating kinase-1 were downregulated after chronic restraint stress stimulation and, subsequently upregulated following treatment with apigenin. Conversely, the levels of mammalian target of rapamycin (mTOR) were increased in chronic restraint stress mice and inhibited by apigenin. Collectively, the present findings indicated that apigenin potentially promotes autophagy via the AMPK/mTOR pathway and induces antidepressive effects in chronic restraint stress mice.
\end{abstract}

\section{Introduction}

Depression results in economically and emotionally over-burdened patients due to the lack of a definitive cure; therapeutic strategies to combat depression are focused on

Correspondence to: Professor Qinan Wu, College of Pharmacy, Nanjing University of Chinese Medicine, 138 Xianlin Road, Nanjing, Jiangsu 210023, P.R. China

E-mail: qnwyjs@163.com

Key words: anti-depression, apigenin, autophagy, chronic restraint stress, adenosine monophosphate-activated protein kinase treating the symptoms $(1,2)$. Selective serotonin reuptake inhibitors (SSRIs) are the most frequently used drugs for the treatment of depression $(3,4)$; however, after long-term clinical observation, the shortcomings of the long-term clinical administration of SSRIs have been noted, such as side effects and delayed efficacy (5-7). Furthermore, $>30 \%$ of patients do not respond strongly to SSRIs (8). Therefore, there is a pressing need for the development of effective drugs to improve depression-like behaviors.

Apigenin is one of the most common flavonoid compounds that are widely distributed in Chinese herbs, such as duckweed and celery $(9,10)$. Previous studies have indicated that apigenin exhibits several pharmacological activities, including antioxidant, anticancer, and anti-inflammatory effects (11-13). In addition, apigenin was found to exert antidepressant effects in chronical unpredictable mild stress- and corticosterone-induced animals $(14,15)$. Evidence indicates that the antidepressant activity of apigenin is partly related to the upregulation of peroxisome proliferator-activated receptor $\gamma$ and brain-derived neurotrophic factor expression levels $(14,15)$. However, the underlying molecular mechanisms of depression are complex and remain to be elucidated.

It is well-known that autophagy is highly associated with the pathogenesis of depressive disorder (16). Autophagy can eliminate damaged organelles and proteins, and is considered to be a conserved process that regulates catabolic processes, and it contributes to the maintenance of cellular energy homeostasis and regulation of cell growth (17). A recent literature review reported that low levels of autophagy have been observed in patients with depression (18). Thus, it is reasonable to hypothesize that normalization of the levels of autophagy may be a potential therapeutic mechanism for the treatment of depression. As indicated in a number of previous studies, apigenin can regulate autophagy in human cancer cell lines $(19,20)$. However, there is little evidence available regarding the underlying mechanisms via which apigenin regulates the levels of autophagy in vivo.

Mammalian target of rapamycin (mTOR)/adenosine monophosphate-activated protein kinase (AMPK) signaling is well known as a classic autophagy-related pathway and is considered to be involved in depression $(21,22)$. The present 
study aimed to clarify the underlying mechanisms by which apigenin might ameliorate depressive-like actions in mice, and hypothesized that apigenin might regulate the activity of AMPK or mTOR, or both. In addition, to access the safety of apigenin, the cytotoxic assay was also performed in vitro. The experimental design is presented in Fig. 1.

\section{Materials and methods}

Animals. A total of 60 male BALB/c mice (age $\sim 6$ weeks, weight $\sim 20 \mathrm{~g}$ ) were purchased from Shanghai SIPPR-BK Laboratory Animal Co., Ltd. (SCXK2013-0016). All animal experiments were approved by the Institutional Animal Care and Use Committee at Nanjing University of Traditional Chinese Medicine, and were conducted in accordance with institutional guidelines for the care and use of laboratory animals. The experimental animals were randomly housed in mouse cages ( 5 mice/cage) for 1 week under the conditions of constant temperature $\left(\sim 23^{\circ} \mathrm{C}\right)$ and humidity $(\sim 50 \%)$, and a 12 -h light/dark cycle, with free access to food and water prior to the experiment.

Establishing the chronic restraint stress model. The chronic restraint stress model was established as previously described (23). Each mouse was single-housed for the whole experimental procedure and placed in a 50-ml centrifuge tube with several ventholes for $6 \mathrm{~h}$ daily (from 9:00 to 15:00) for 3 weeks. Following chronic restraint stressing, the mice were returned to their original cages. Additionally, overnight illumination was randomly performed on all mice twice-weekly. The mice in the control group were group-housed under standard conditions $(\mathrm{n}=8)$. The body weights of all mice were recorded every week.

Drugs and administration. Apigenin was obtained from Jiangsu Collaborative Innovation Center of Chinese Medicinal Resources Industrialization (Nanjing University of Traditional Chinese Medicine), and subsequently dissolved in normal saline with $0.5 \% \mathrm{w} / \mathrm{v}$ Tween- 80 prior to administration via gavage (20, 40 and $60 \mathrm{mg} / \mathrm{kg}$; n=10/group). Further, $25 \mathrm{mg} / \mathrm{kg}$ fluoxetine hydrochloride (Tokyo Chemical Industry Co., Ltd.) was dissolved in normal saline and administered via gavage $(n=10)$. The mice in the control group were treated with normal saline with $0.5 \% \mathrm{w} / \mathrm{v}$ Tween- 80 via gavage $(\mathrm{n}=8)$. The administration was started on the 22th day of modeling (modeling was carried out from the day one) and lasted for 14 days (once a day). The drug doses were optimized according to our previous optimal dose-response-relationship studies (data not shown).

Sucrose preference test. The sucrose preference test was performed every 7 days throughout the experimental period. Mice were single-housed, and then each mouse was presented with two bottles filled with $2 \%$ sucrose water for 3 consecutive days. After an 18-h deprivation of both water and food, each mouse was presented with two bottles for $2 \mathrm{~h}$ with the same appearance: One filled with clear water and the other with $2 \%$ sucrose water. Sucrose preference was calculated using the following formula: Sucrose preference $(\%)=$ sucrose solution consumption (g)/total consumption (g).
Open field test. The anxiety-like behavior and physical condition of the mice were analyzed in the open field test, which was conducted as described previously (24). On the 36th day of the experimental process, each mouse was subjected to the open field test in a bright open area $(\sim 300$ lux, $40 \times 40 \mathrm{~cm})$. The mice were softly placed in the test area and allowed to explore freely for $5 \mathrm{~min}$. Digitized images of the active orbit of each mouse were recorded. The total distance travelled and the time spent in the center were analyzed using ANY-maze software (version 4.3; Stoelting Co.) to evaluate the locomotor activity and anxiety-like behavior of mice. The experimental apparatus was washed with $70 \%$ ethyl alcohol between consecutive tests.

Forced swim test. Following a 2 -week intervention administration, each mouse were gently placed into a 5-1 cylindrical transparent glass tank with clear water $\left(\sim 23^{\circ} \mathrm{C}\right)$ and forced to swim for $6 \mathrm{~min}$. The immobility time was measured during the final $4 \mathrm{~min}$ of the $6 \mathrm{~min}$ from the video recorded using ANY-maze software. Following the test, the mice were dried with an electric hair dryer and returned to their cages.

Tail suspension test. The tail suspension test is employed to evaluate depressive-like behavior and the response to antidepressant treatments in mice at day 36 (25). The test was performed by an ANY-maze system that recorded 6 animals at a time. Each mouse was suspended by the tail at $50 \mathrm{~cm}$ above the floor with adhesive tape affixed $1 \mathrm{~cm}$ from the tip of the tail. The entire test required 6 min to complete, and animals were considered to be mobile or immobile. The final $4 \mathrm{~min}$ of the total 6 min were analyzed using ANY-maze software to quantify the immobility time.

Western blot analysis. All mice were euthanized by cervical dislocation after the behavioral tests, and the hippocampus samples were promptly collected and placed on ice. The samples were then placed into radioimmunoprecipitation assay buffer (Beyotime Institute of Biotechnology) with an enzyme inhibitor (Beyotime Institute of Biotechnology) at $5^{\circ} \mathrm{C}$ and rapidly homogenized for western blotting, as previously reported $(21,26)$. Protein concentration was determined colorimetrically by BCA assay (Pierce; Thermo Fisher Scientific, Inc.). Protein lysates $(30 \mu \mathrm{g})$ were separated by $10 \%$ SDS-PAGE electrophoresis and were transferred onto polyvinylidene difluoride (PVDF) membranes. The primary antibodies, incubated at $4^{\circ} \mathrm{C}$ for $12 \mathrm{~h}$, included: Rabbit anti-AMPKa $(1: 1,000 ; 2532 S)$, rabbit anti-phosphorylated (p)-AMPKa (Thr172; 1:1,000; 2535S), anti-p-Unc-51 like autophagy activating kinase-1 (ULK1; Ser317; 1:1000; 12753S), rabbit anti-p-mTOR $(1: 1,000 ; 2971 \mathrm{~S})$, rabbit anti-mTOR $(1: 1,000$; 2792S; all obtained from Cell Signaling Technology, Inc.), rabbit anti-microtubule-associated protein light chain 3 (LC3)-II/I (1:1,000, 14600-1-AP), rabbit anti-ULK1 (1:1,000; 20986-1-AP), rabbit anti-p62/sequestosome 1 (SQSTM1; $1: 1,000 ; 18420-1-A P)$ and rabbit anti-GAPDH $(1: 3,000$; 10494-1-AP; all purchased from ProteinTech Group, Inc.). The secondary antibody, incubated at room temperature for $2 \mathrm{~h}$, was horseradish peroxidase-conjugated goat anti-rabbit immunoglobulin G (1:3,000; SA00001-2; ProteinTech 


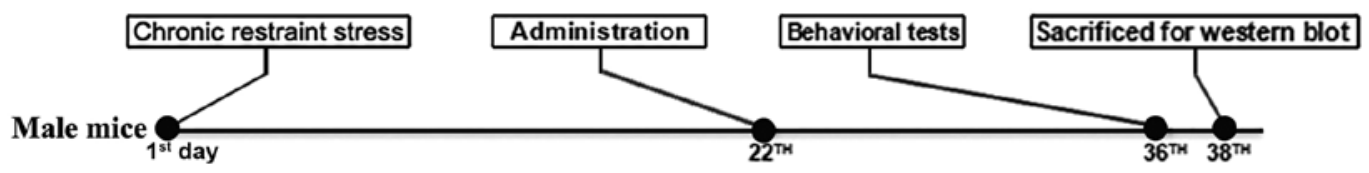

Figure 1. Schedule of the study. Mice were subjected to 2 weeks' administration of vehicle, fluoxetine or apigenin (20, 40 or 60 mg/kg) following 21 days stress. On the 36th day, behavioral tests were conducted on each mouse. Subsequently, the mice were euthanized, and the hippocampal tissues were removed to analyze the protein expression levels.

A

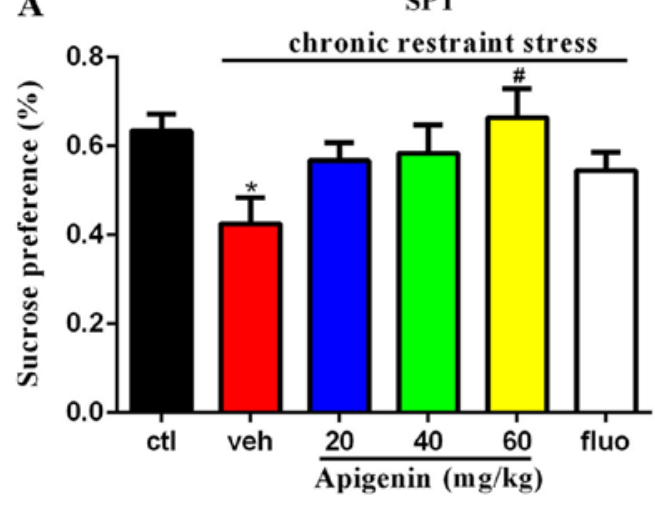

B

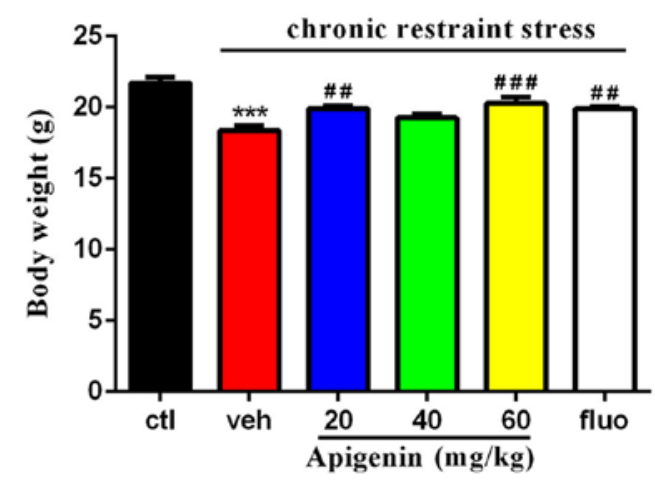

Figure 2. Apigenin treatment induces increased preference for sucrose and body weight. (A) 2-week treatment with apigenin or fluoxetine improved deficits in sucrose preference in chronic restraint stress mice ( $\mathrm{n}=7-8 /$ group). (B) Body weight was increased by apigenin ( $\mathrm{n}=8-9 /$ group). ${ }^{*} \mathrm{P}<0.05$, ${ }^{* * * *} \mathrm{P}<0.001$ vs. ctl; ${ }^{\#} \mathrm{P}<0.05,{ }^{\#} \mathrm{P}<0.01,{ }^{\# \# \#} \mathrm{P}<0.001$ vs. veh. SPT, sucrose preference test; ctl, control group; veh, vehicle group; fluo, fluoxetine group.

Group, Inc.). Densitometry was conducted and analyzed using ImageJ software (version $1.52 \mathrm{a}$, National Institutes of Health). Blots were visualized using a SuperSignal West Pico Chemiluminescent Substrate (Thermo Fisher Scientific, Inc.). The loading amounts in each lane were normalized to GAPDH. All experiments were performed three times.

Cytotoxicity assay. For cytotoxicity assays, cells were grown in Dulbecco's Modified Eagle's medium (Gibco; Thermo Fisher Scientific Inc.) with $10 \%$ fetal bovine serum (Gibco; Thermo Fisher Scientific Inc.) in an atmosphere of 5\% $\mathrm{CO}_{2}$ and $95 \%$ air for $24 \mathrm{~h}$ at $37^{\circ} \mathrm{C}$. HT22, SH-SY5Y and N2a cells (obtained from Shanghai Zhong Qiao Xin Zhou Biotechnology Co., Ltd.) were seeded into 60 wells of a 96 -well plate $(6,000$ cells/well), with the remaining wells holding media. Following incubation for $24 \mathrm{~h}$, cells were treated with various concentrations of apigenin $(0,3.125,6.25,12.5,25,50$ and $100 \mu \mathrm{M}$ dissolved in $0.1 \%$ DMSO) for $48 \mathrm{~h}$. Thereafter, MTT was added into each well with a volume of $20 \mu \mathrm{l}$ and incubated for $4 \mathrm{~h}$. DMSO was added to each well and the absorbance was measured at $492 \mathrm{~nm}$ using a microplate reader (Thermo Fisher Scientific, Inc.). The $\mathrm{CC}_{50}$ values were calculated as the concentration of apigenin resulting in $50 \%$ reduction of absorbance compared to untreated cells. All experiments were performed three times.

Statistical analysis. All data were expressed as the mean \pm standard error of the mean. Differences among groups were analyzed using one-way ANOVA with Bonferroni correction to adjust for multiple testing, and $\mathrm{P}<0.05$ was considered to indicate a statistically significant difference. Statistical analysis was performed with GraphPad Prism 6.0 software (GraphPad Software, Inc.).

\section{Results}

Apigenin improves the deficits in sucrose preference and body weight. A significant difference in sucrose preference was observed in the chronic restraint stress group (vehicle group) compared with the control group ( $\mathrm{P}<0.05$; Fig. 2A). A significant effect of treatment on sucrose preference was observed $\left(\mathrm{F}_{(5,39)}=2.646, \mathrm{P}=0.0375\right)$; as presented in Fig. $2 \mathrm{~A}$, the deficits induced by chronic restraint stress were significantly ameliorated following treatment with $60 \mathrm{mg} / \mathrm{kg}$ apigenin for 14 days $(\mathrm{P}<0.05$ vs. vehicle group). Additionally, a significant effect of treatment on body weight was observed $\left(\mathrm{F}_{(5,44)}=11.55\right.$, $\mathrm{P}<0.0001$ ); specifically, both 20 and $60 \mathrm{mg} / \mathrm{kg}$ apigenin significantly increased body weight compared with the vehicle group (20 mg/kg, P<0.01; 60 mg/kg, P<0.001; Fig. 2B).

Effects of apigenin on the center time in the open field test. In the open field test (Fig. 3A), the time spent in the center of the experimental area was not significantly affected by treatment group $\left(F_{(5,31)}=2.014, P=0.1041\right)$; however, all doses of apigenin demonstrated a non-significant trend towards prolonging the time spent in the center, which was markedly reduced by chronic restraint stress. The total distance travelled (Fig. 3B) was significantly affected by animal treatment $\left(\mathrm{F}_{(5,42)}=3.684, \mathrm{P}=0.0075\right)$; however, no significant differences were observed between the control and vehicle groups, or the vehicle and apigenin groups $(\mathrm{P}>0.05)$. Reduced time spent in the center is indicative of anxiety-like behavior in mice, and the total distance indicated that apigenin or chronic restraint stress did not alter the motor ability of mice.

Apigenin decreases the immobility time in both the forced swim test and tail suspension test. As presented in Fig. 4, treatment significantly affected the immobility time in the 
A

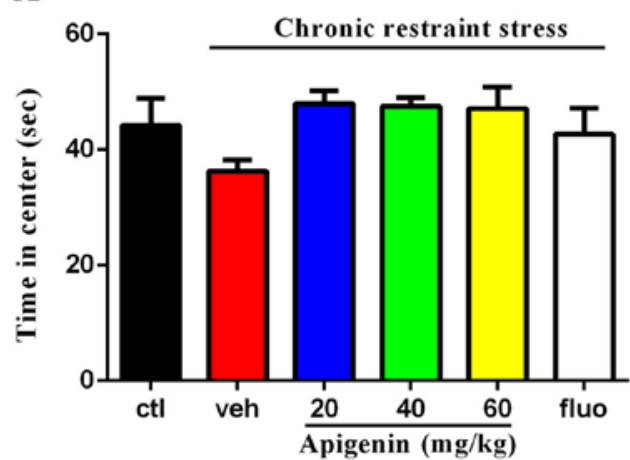

B

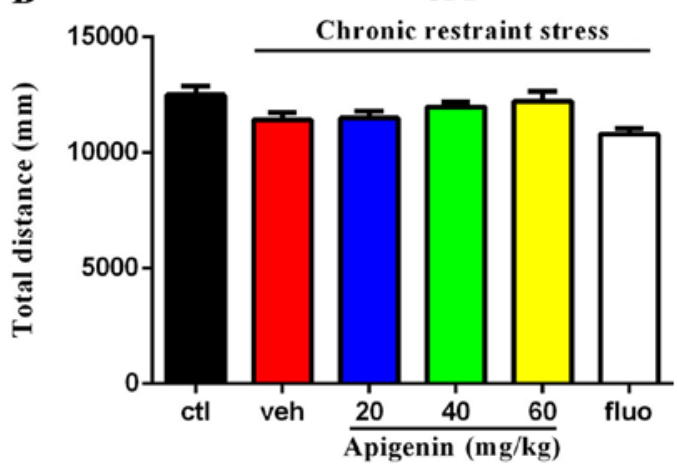

Figure 3. Effects of chronic restraint stress and apigenin on mouse behavior in the open field test. (A) Chronic restraint stress mice spent reduced time in the center in the OFT (n=6-7/group). (B) No significant difference was observed in the total distance among groups (n=8/group). OFT, open field test; ctl, control group; veh, vehicle group; fluo, fluoxetine group.

A

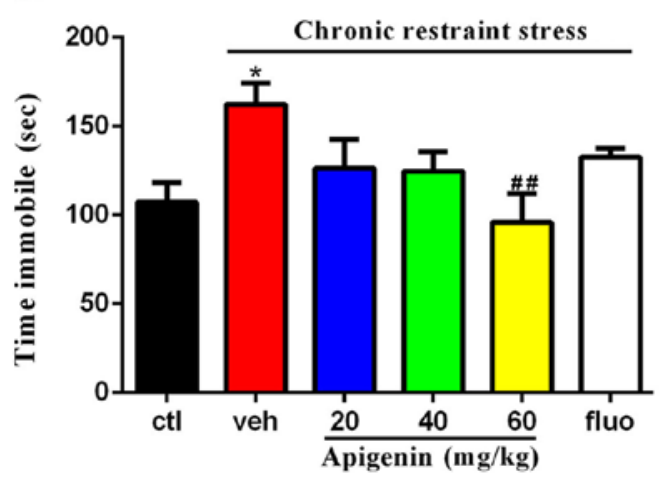

B

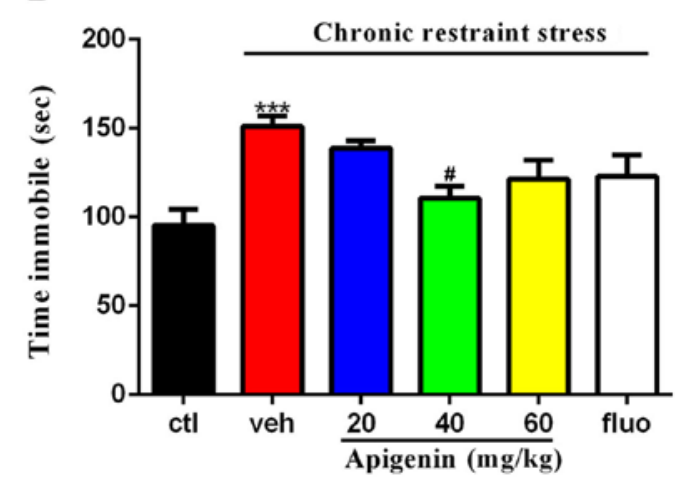

Figure 4. Apigenin decreases the immobility time in the TST and FST. (A) Apigenin significantly reduced the immobility time in the TST (n=7-8/group). (B) Deficits in the FST were also improved by apigenin ( $\mathrm{n}=7-8 /$ group). ${ }^{*} \mathrm{P}<0.05,{ }^{* * *} \mathrm{P}<0.001$ vs. ctl; ${ }^{*} \mathrm{P}<0.05$, ${ }^{\# \#} \mathrm{P}<0.01$ vs. veh. TST, tail suspension test; FST, forced swim test; ctl, control group; veh, vehicle group; fluo, fluoxetine group.

tail suspension $\left(\mathrm{F}_{(5,37)}=3.162, \mathrm{P}=0.0178\right)$ and forced swim tests $\left(\mathrm{F}_{(5,39)}=5.282, \mathrm{P}=0.0008\right)$. The prolonged immobility time induced by chronic restraint stress $(\mathrm{P}<0.05$ vs. control group) in the tail suspension test was rescued by the administration of $60 \mathrm{mg} / \mathrm{kg}$ apigenin ( $\mathrm{P}<0.01$ vs. vehicle group; Fig. 4A). In the forced swim test (Fig. 4B), the increased immobility time induced by chronic restraint stress $(\mathrm{P}<0.001$ vs. control group) was significantly reduced following $40 \mathrm{mg} / \mathrm{kg}$ apigenin treatment ( $\mathrm{P}<0.05$ vs. vehicle group). The alleviation of these behavioral test deficits indicated the antidepressive actions of apigenin in depressive-like mice.

Apigenin regulates the degree of autophagy in hippocampus. The expression levels of LC3-II/I and p62/SQSTM1 were assessed in order to measure the degree of autophagy in the hippocampus; treatment significantly affected the expression of LC3-II/ $\left(\mathrm{F}_{(4,22)}=5.621, \mathrm{P}=0.0028\right)$ and p62 $\left(\mathrm{F}_{(4,17)}=7.041\right.$, $\mathrm{P}=0.0016$; Fig. 5). As presented in Fig. 5, the levels of autophagy were found to be reduced in the vehicle group, as determined by the significantly downregulated expression of LC3-II/I ( $\mathrm{P}<0.01$ vs. control group) and increased level of p62/SQSTM1 ( $\mathrm{P}<0.05$ vs. control group). Conversely, apigenin significantly enhanced the levels of LC3-II $/ \mathrm{I}(20 \mathrm{mg} / \mathrm{kg}$, $\mathrm{P}<0.05 ; 40$ and $60 \mathrm{mg} / \mathrm{kg}, \mathrm{P}<0.01 \mathrm{vs}$. vehicle group) and downregulated the expression of p62 $(20 \mathrm{mg} / \mathrm{kg}, \mathrm{P}<0.01 ; 40 \mathrm{mg} / \mathrm{kg}$,
$\mathrm{P}<0.05 ; 60 \mathrm{mg} / \mathrm{kg}, \mathrm{P}<0.001$ vs. vehicle group) in hippocampal samples. These findings indicated that apigenin regulated the degree of autophagy in chronic restraint stress mice.

Apigenin activity is mediated via AMPK/ULK1 signaling. Treatment significantly affected the phosphorylation of AMPK $\left(\mathrm{F}_{(4,21)}=4.298, \mathrm{P}=0.0107\right.$; Fig. 6A); the expression levels of p-AMPK were rescued following administration of apigenin ( $20 \mathrm{mg} / \mathrm{kg}, \mathrm{P}<0.01 ; 60 \mathrm{mg} / \mathrm{kg}, \mathrm{P}<0.05$ vs. vehicle group). ULK1-Ser317 levels were not significantly affected by treatment $\left(\mathrm{F}_{(4,15)}=2.907, \mathrm{P}=0.0578\right.$; Fig. 6B). Fig. 6C and D present the significant effects of treatment on the p-AMPK/AMPK $\left(\mathrm{F}_{(4,21)}=17.83, \mathrm{P}<0.0001\right.$; Fig. 6C) and ULK1-Ser317/ULK1 ratios $\left(\mathrm{F}_{(4,15)}=8.596, \mathrm{P}=0.0008\right.$; Fig. 6D). The decreased levels of $\mathrm{p}$-AMPK/AMPK ( $\mathrm{P}<0.001$ vs. control group) and ULK1-Ser317/ULK1 ( $\mathrm{P}<0.05$ vs. control group) induced by chronic restraint stress were significantly reversed following the administration of apigenin (all doses, $\mathrm{P}<0.001$ vs. vehicle group; Fig. 6C; $60 \mathrm{mg} / \mathrm{kg}, \mathrm{P}<0.05$ vs. vehicle group; Fig. 6D). The results indicated that the AMPK/ULK1 pathway may be a target of apigenin in the hippocampus of depressive-like mice.

Phosphorylation of mTOR is inhibited by apigenin. The phosphorylation of mTOR normalized to GAPDH $\left(\mathrm{F}_{(4,22)}=7.425\right.$, $\mathrm{P}=0.0006)$ and total mTOR $\left(\mathrm{F}_{(4,22)}=10.75, \mathrm{P}<0.0001\right)$ was 

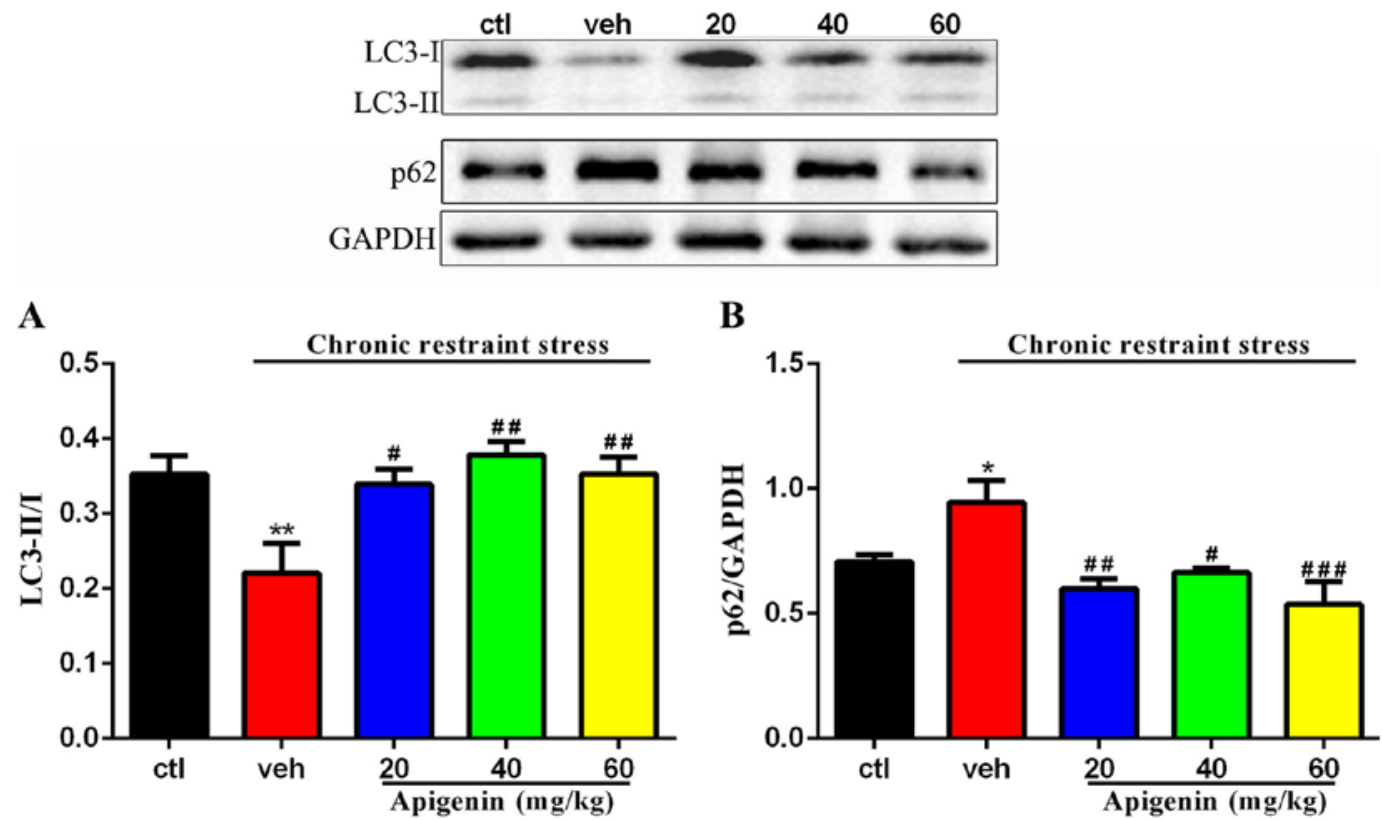

Figure 5. Hippocampal p62 and LC3-II/I are regulated by apigenin. (A) Expression levels of LC3-II/I were increased after administration of apigenin (LC3-II/I $=\frac{\mathrm{LC} 3-\mathrm{II} / \mathrm{GAPDH}}{\mathrm{LC3}-\mathrm{I} / \mathrm{GAPDH}}, \mathrm{n}=5-6 /$ group). (B) Levels of $\mathrm{p} 62$ were significantly increased in chronic restraint stress mice, and significantly reduced following apigenin treatment (p62 was normalized to GAPDH, n=4-5/group). ${ }^{*} \mathrm{P}<0.05,{ }^{* *} \mathrm{P}<0.01$ vs. ctl; ${ }^{\# \mathrm{P}}<0.05,{ }^{\# \#} \mathrm{P}<0.01,{ }^{\# \# \#} \mathrm{P}<0.001$ vs. veh. LC, microtubule-associated protein light chain; ctl, control group; veh, vehicle group.
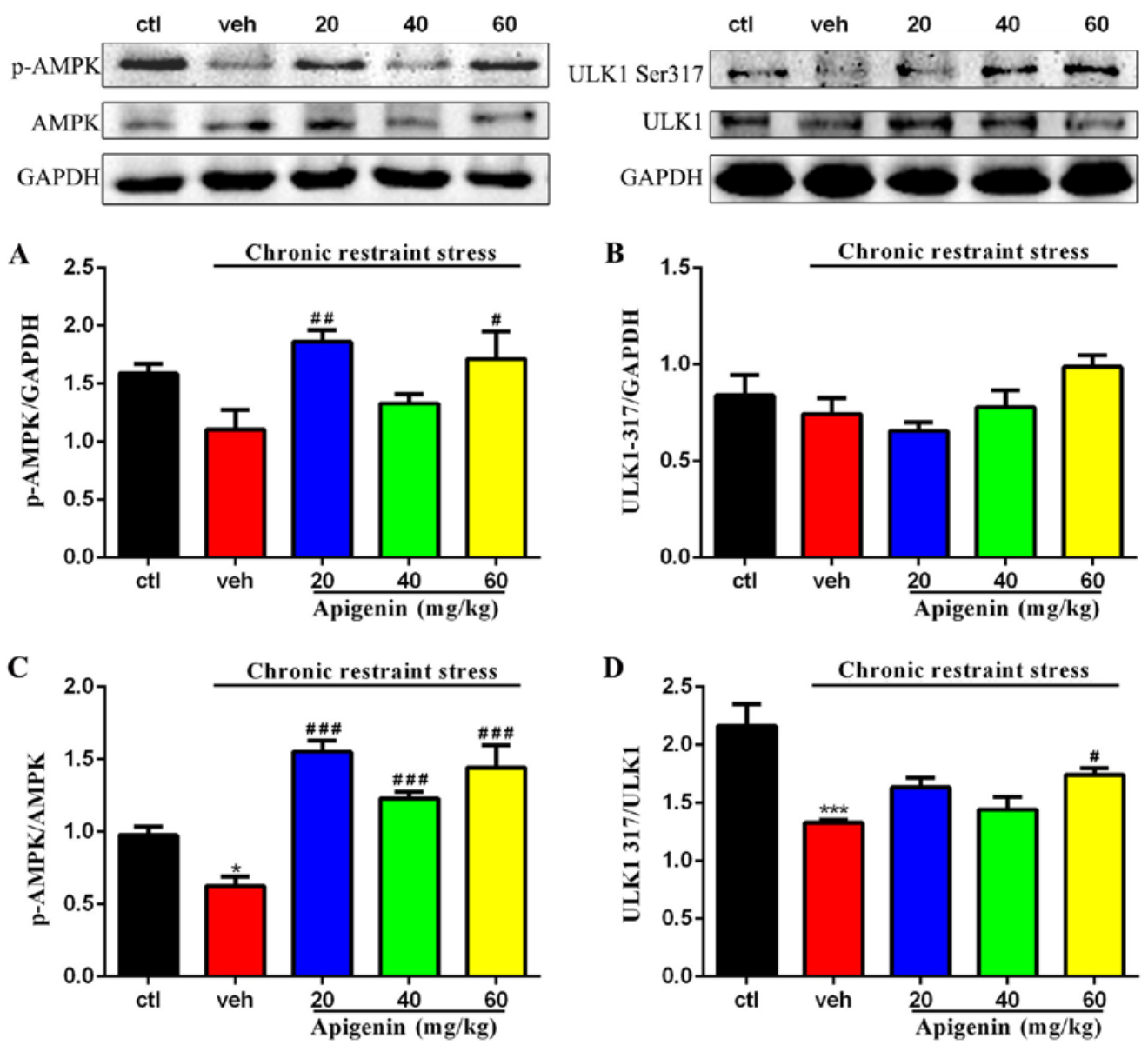

Figure 6. AMPK-ULK1 pathway is regulated by apigenin in the hippocampus. (A) Expression of p-AMPK was promoted by apigenin (p-AMPK was normalized to GAPDH, n=5-6/group). (B) Apigenin activated ULK1-Ser317 signaling (ULK1-Ser317 was normalized to GAPDH, n=3-5/group). (C) Expression of p-AMPK/AMPK was significantly upregulated by apigenin (p-AMPK/AMPK= $\frac{\text { P-AMPK/GAPDH }}{\text { AMPK/GAPDH }}, \mathrm{n}=5 /$ group). (D) Low level of ULK1-Ser317/ULK1 induced by

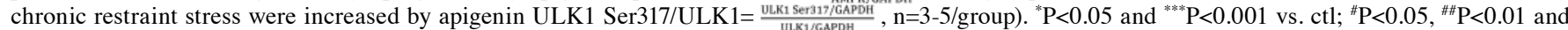

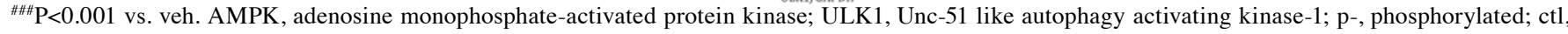
control group; veh, vehicle group. 


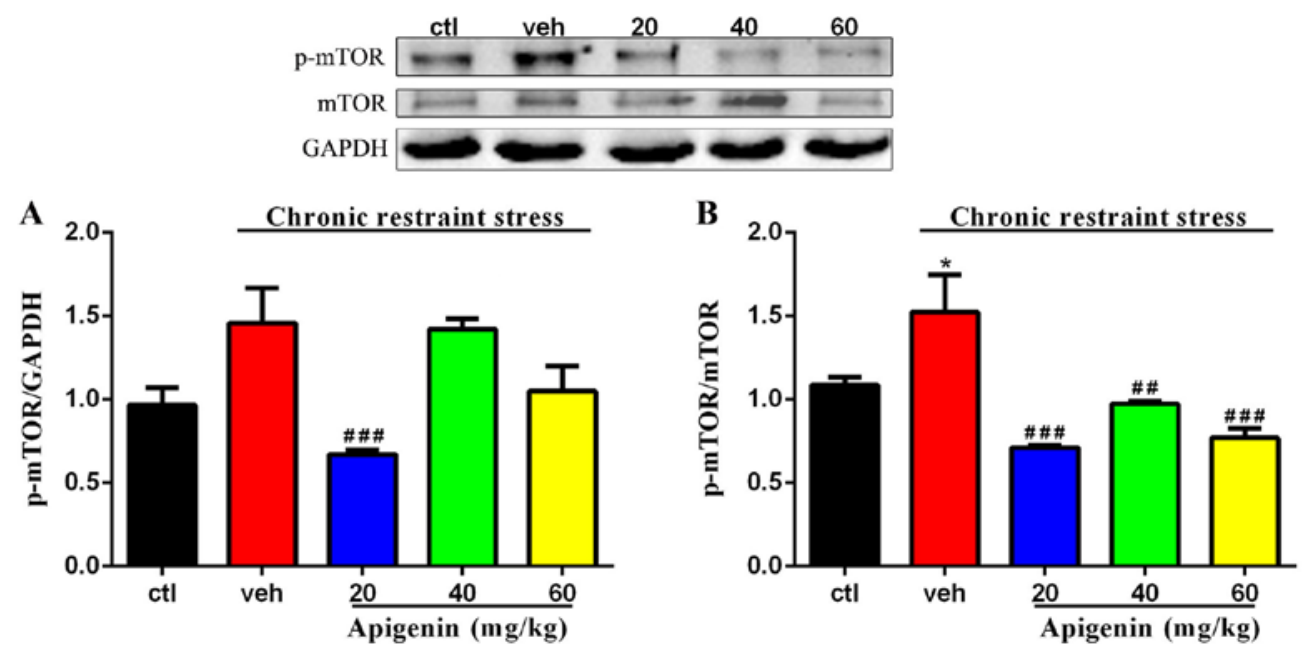

Figure 7. mTOR signaling is regulated by apigenin in the hippocampus. (A) Expression of p-mTOR was increased in chronic restraint stress mice (p-mTOR

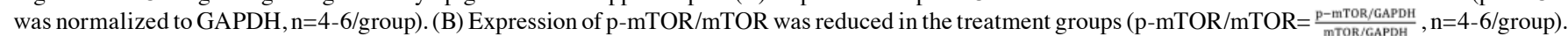
"P<0.05 vs. ctl; ${ }^{\# \#} \mathrm{P}<0.01,{ }^{\# \# \#} \mathrm{P}<0.001$ vs. veh. mTOR, mammalian target of rapamycin; $\mathrm{p}$-, phosphorylated; ctl, control group; veh, vehicle group.
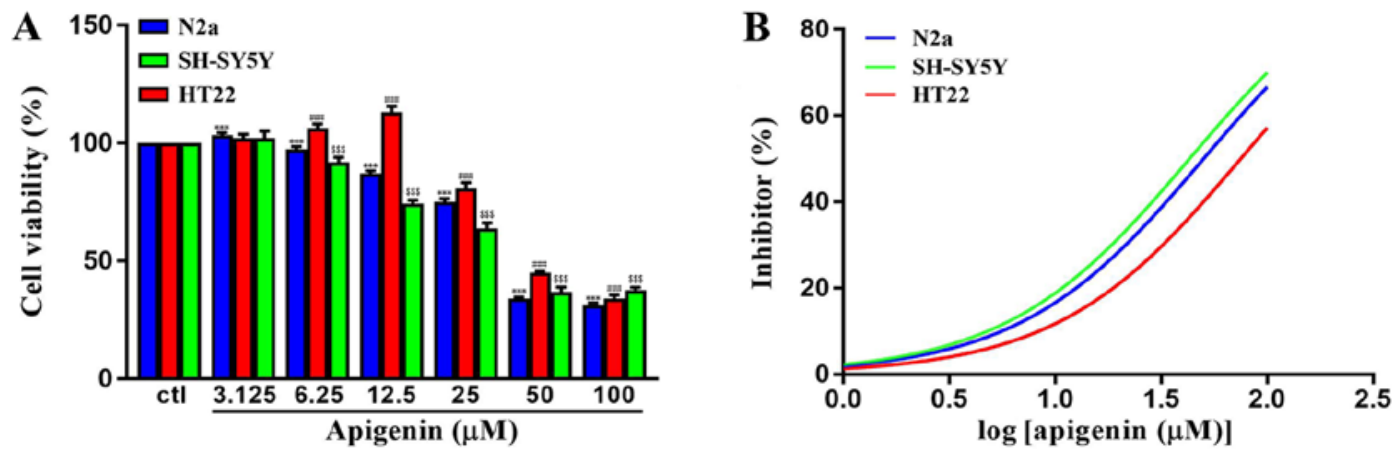

Figure 8. Apigenin exerts cytotoxic effects at high concentrations in vitro. (A) Decreased cell viability was observed in SH-SY5Y, HT22 and N2a cells following 48-h treatment with apigenin at high concentrations ( $\mathrm{n}=8 /$ group). (B) Dose-response inhibition curves revealed the inhibition of different cell lines following exposure to apigenin $(0,3.125,6.25,12.5,25,50$ and $100 \mu \mathrm{M})$ for $48 \mathrm{~h}$. The rate of inhibition=(100-cell viability $) \%$. ${ }^{* * *} \mathrm{P}<0.001,{ }^{\# \# \#} \mathrm{P}<0.001$, ${ }^{\$ \$ \$} \mathrm{P}<0.001$ vs. respective ctl groups. ctl, control group.

significantly affected by treatment group (Fig. 7). As presented in Fig. 7A, the increase in the levels of p-mTOR induced by chronic restraint stress in the vehicle group was significantly reversed following treatment with apigenin $(20 \mathrm{mg} / \mathrm{kg}, \mathrm{P}<0.001$ vs. vehicle group). The expression levels of $\mathrm{p}-\mathrm{mTOR} / \mathrm{mTOR}$ were significantly increased by chronic restraint stress $(\mathrm{P}<0.05$ vs. control group; Fig. 7B); however, following administration of apigenin, the $\mathrm{p}-\mathrm{mTOR} / \mathrm{mTOR}$ ratio was significantly reduced ( 20 and $60 \mathrm{mg} / \mathrm{kg}, \mathrm{P}<0.001 ; 40 \mathrm{mg} / \mathrm{kg}, \mathrm{P}<0.01$ vs. vehicle group). These results suggest that the activity of mTOR was involved in apigenin-mediated autophagy level.

Cytotoxic effects of apigenin in vitro. To determine the cytotoxic potential of apigenin, its effects on the viability of different cell lines were evaluated (Fig. 8A). Apigenin induced a significant increase in the viability of HT22 cells at concentrations of $\leq 12.5 \mu \mathrm{M}(\mathrm{P}<0.001)$; however, it induced cytotoxic effects in all cell lines at high concentrations $\left(\mathrm{F}_{(6,49)}=8,653\right.$, $\mathrm{P}<0.0001$ for $\mathrm{N} 2 \mathrm{a} ; \mathrm{F}(6,49)=2,445, \mathrm{P}<0.0001$ for $\mathrm{HT} 22$; $\mathrm{F}_{(6,49)}=1,533, \mathrm{P}<0.0001$ for SH-SY5Y). As presented in Fig. 8B, it was revealed that the $\mathrm{CC}_{50}$ of apigenin in SH-SY5Y, HT22 and $\mathrm{N} 2 \mathrm{a}$ cells were 42.97, 74.96 and $50.06 \mu \mathrm{M}$, respectively.
The data indicated that apigenin exerted dose-dependent cytotoxic effects on neuronal cells.

\section{Discussion}

Apigenin is a natural product in various Chinese herbs and exhibits high bioactivity, including anticancer, antiinflammatory, and antifibrotic effects (27-29). In addition, previous studies have indicated that it also elicits neuroprotective effects $(30,31)$. In the present study, depressive-like mice, induced by chronic restraint stress, were used to investigate the antidepressant effects of apigenin. Chronic restraint stress has become a widely employed rodent model for depression-like disorders (23,32-34). Fluoxetine was used as a positive control drug, and, similar to fluoxetine, apigenin was observed to increase sucrose preference and decrease the immobility time in behavioral tests. According to the cytotoxicity assay, apigenin is a safe and easily-accessed compound, widely used in various Chinese herbs and food. The findings of the present study suggested that apigenin can exert antidepressive effects in chronic restraint stress model mice. 
Clinically, the degree of autophagy has been reported to be significantly reduced in the sera and brains of patients with depression $(35,36)$. Autophagy has been demonstrated to be closely associated with the pathogenesis of various neurological disorders or conditions, including Alzheimer's and Parkinson's disease, and depression (37-39). LC3-II/I and p62 have been confirmed to be relevant biomarkers of upregulated autophagy (40). In the present study, low levels of autophagy, as determined by these two biomarkers, were observed in depressive-like mice, but were attenuated by apigenin treatment, indicating that apigenin is capable of promoting autophagy. This result suggested that the antidepressant effects of apigenin in chronic restraint stress mice were due to its ability to regulate autophagy.

mTOR/AMPK/ULK1 signaling has been demonstrated to be a crucial pathway associated with autophagy (41). ULK1 initiates autophagic processes, and AMPK directly phosphorylates ULK1 at Ser317 to promote autophagy (42). mTOR complex 1 also serves a crucial role in the molecular processes of autophagy (43). Compelling evidence indicates that mTOR inhibits autophagy, and that rapamycin, which suppresses the activity of mTOR, can block this action (44). mTOR competitively binds ULK1 at Ser757 and inhibits autophagy, disturbing the connection between AMPK and ULK1 (45). The present study revealed elevated levels of p-mTOR in the hippocampus of depressive-like mice. The two-week administration of apigenin significantly modulated the activity of mTOR. According the results of the present study, the responses of ULK1 and AMPK to stress and apigenin treatment were opposite to that of $\mathrm{mTOR}$, indicating that apigenin may regulate autophagy via mTOR/AMPK/ULK1 signaling.

In conclusion, the results of the present study suggested that apigenin exerts antidepressant effects on chronic restraint stress mice and promotes autophagy by regulating mTOR/AMPK/ULK1 signaling. The present study preliminarily revealed the potential mechanisms of apigenin; however, further metabonomic, proteomic and transcriptomic studies are required as next steps in the development of apigenin as a therapeutic agent for the treatment of depression.

\section{Acknowledgements}

Not applicable.

\section{Funding}

The authors gratefully acknowledge the financial support of Chinese National Natural Science Foundation (grant no. 81073002); The National '25-Year' Technology Support Program (grant no. 2011BAI04B06); The Program of Collaborative Innovation Center of Chinese Medicinal Material Resources Industrialization of Jiangsu Province (2016); and Postgraduate Education Reform Project of Jiangsu Province (grant nos. KYCX18_1601; KYCX18_1628).

\section{Availability of data and materials}

The datasets used and/or analyzed in the current study are available from the corresponding author upon reasonable request. The role of the funding body in the design of the study and collection, analysis, and interpretation of data and in writing the article should be declared in this request.

\section{Authors' contributions}

QW and XZ contributed to the concept and design of the present study. XZ. XH, ZH, HB, YJ, GS ,RJ was involved in data acquisition, analysis and interpretation. $\mathrm{XZ}$ and HD drafted and critically revised the article for important intellectual content.

\section{Ethics approval and consent to participate}

All animal experiments were approved by the Institutional Animal Care and Use Committee at Nanjing University of Traditional Chinese Medicine.

\section{Patient consent for publication}

Not applicable.

\section{Competing interests}

The authors declare that they have no competing interests.

\section{References}

1. Steel N, Ford JA, Newton JN, Davis ACJ, Vos T, Naghavi M, Glenn S, Hughes A, Dalton AM, Stockton D, et al: Changes in health in the countries of the UK and 150 English local authority areas 1990-2016: A systematic analysis for the Global Burden of Disease Study 2016. Lancet 392: 1647-1661, 2018.

2. Ogbo FA, Mathsyaraja S, Koti RK, Perz J and Page A: The burden of depressive disorders in South Asia, 1990-2016: Findings from the global burden of disease study. BMC Psychiatry 18: 333, 2018.

3. Castellano S, Ventimiglia A, Salomone S, Ventimiglia A, De Vivo S, Signorelli MS, Bellelli E, Santagati M, Cantarella RA, Fazio E, et al: Selective serotonin reuptake inhibitors and serotonin and noradrenaline reuptake inhibitors improve cognitive function in partial responders depressed patients: Results from a prospective observational cohort study. CNS Neurol Disord Drug Targets 15: 1290-1298, 2016.

4. Vaswani M, Linda FK and Ramesh S: Role of selective serotonin reuptake inhibitors in psychiatric disorders: A comprehensive review. Prog Neuropsychopharmacol Biol Psychiatry 27: 85-102, 2003.

5. Stahl SM: Mechanism of action of serotonin selective reuptake inhibitors. Serotonin receptors and pathways mediate therapeutic effects and side effects. J Affect Disord 51: 215-235, 1998

6. Nørr L, Bennedsen B, Fedder J and Larsen ER: Use of selective serotonin reuptake inhibitors reduces fertility in men. Andrology 4: 389-394, 2016.

7. Li YJ and Li YM: Gestational exposure to selective serotonin reuptake inhibitors and offspring psychiatric disorders: Need for further investigation. J Am Acad Child Adolesc Psychiatry 55: 726, 2016

8. Charney DS, Grothe DR, Smith SL, Brady KT, KaltsounisPuckett J, Wright CW, Laird LK and Rush AJ: Overview of psychiatric disorders and the role of newer antidepressants. J Clin Psychiatry 63 (Suppl 1): S3-S9, 2002.

9. Barros GO, Woodard SL and Nikolov ZL: Phenolics removal from transgenic Lemna minor extracts expressing mAb and impact on mAb production cost. Biotechnol Prog 27: 410-418, 2011.

10. Tan GF, Ma J, Zhang XY, Xu ZS and Xiong AS: AgFNS overexpression increase apigenin and decrease anthocyanins in petioles of transgenic celery. Plant Sci 263: 31-38, 2017.

11. Nabavi SF, Khan H, D'Onofrio G, Samec D, Shirooie S, Dehpour AR, Argüelles S, Habtemariam S and SobarzoSanchez E: Apigenin as neuroprotective agent: Of mice and men. Pharmacol Res 128: 359-365, 2018. 
12. Li F, Lang F, Zhang H, Xu L, Wang Y, Zhai C and Hao E: Apigenin alleviates endotoxin-induced myocardial toxicity by modulating inflammation, oxidative stress, and autophagy. Oxid Med Cell Longev 2017: 2302896, 2017.

13. Soyman Z, Kelekçi S, Sal V, Şevket O, Bayindir N and Uzun H: Effects of Apigenin on experimental Ischemia/Reperfusion injury in the rat ovary. Balkan Med J 34: 444-449, 2017.

14. Li R, Wang X, Qin T, Qu R and Ma S: Apigenin ameliorates chronic mild stress-induced depressive behavior by inhibiting interleukin-1 $\beta$ production and NLRP3 inflammasome activation in the rat brain. Behav Brain Res 296: 318-325, 2016.

15. Weng L, Guo X, Li Y, Yang X and Han Y: Apigenin reverses depression-like behavior induced by chronic corticosterone treatment in mice. Eur J Pharmacol 774: 50-54, 2016.

16. Tan X, Du X, Jiang Y, Botchway BOA, Hu Z and Fang M: Inhibition of autophagy in microglia alters Depressive-like behavior via BDNF pathway in postpartum depression. Front Psychiatry 9: 434, 2018.

17. Anding AL and Baehrecke EH: Autophagy in cell life and cell death. Curr Top Dev Biol 114: 67-91, 2015.

18. Muller S, Brun S, René F, de Séze J, Loeffler JP and Jeltsch-David H: Autophagy in neuroinflammatory diseases. Autoimmun Rev 16: 856-874, 2017.

19. Salmani JMM, Zhang XP, Jacob JA and Chen BA: Apigenin's anticancer properties andmolecular mechanisms of action: Recent advances and future prospectives. Chin J Nat Med 15: 321-329, 2017.

20. Yang J, Pi C and Wang G: Inhibition of PI3K/Akt/mTOR pathway by apigenin induces apoptosis and autophagy in hepatocellular carcinoma cells. Biomed Pharmacother 103: 699-707, 2018.

21. Huang Z, Huang X, Wang Q, Jiang R, Sun G, Xu Y and Wu Q: Extract of Euryale ferox Salisb exerts antidepressant effects and regulates autophagy through the adenosine monophosphate-activated protein kinase-UNC-51-like kinase 1 pathway. IUBMB Life 70: 300-309, 2018.

22. Huang X, Wu H, Jiang R, Sun G, Shen J, Ma M, Ma C, Zhang S, Huang Z, Wu Q, et al: The antidepressant effects of a-tocopherol are related to activation of autophagy via the AMPK/mTOR pathway. Eur J Pharmacol 833: 1-7, 2018.

23. Kim YR, Park BK, Kim YH, Shim I, Kang IC and Lee MY Antidepressant Effect of Fraxinus rhynchophylla hance extract in a mouse model of chronic Stress-induced depression. Biomed Res Int 2018: 8249563,2018.

24. Xue W, Wang W, Gong T, Zhang H, Tao W, Xue L, Sun Y, Wang $F$ and Chen G: PKA-CREB-BDNF signaling regulated long lasting antidepressant activities of Yueju but not ketamine. Sci Rep 6: 26331, 2016.

25. Castagné V, Moser P, Roux S and Porsolt RD: Rodent models of depression: Forced swim and tail suspension behavioral despair tests in rats and mice. Curr Protoc Neurosc: Chapter 8: Unit 8.10A, 2011. doi: 10.1002/0471142301.ns0810as55.

26. Zhang S, Xu S, Duan H, Zhu Z, Yang Z, Cao J, Zhao Y, Huang Z, Wu Q and Duan J: A novel, highly-water-soluble apigenin derivative provides neuroprotection following ischemia in male rats by regulating the ERK/Nrf2/HO-1 pathway. Eur J Pharmacol 855: 208-215, 2019

27. Zhang J, Chao L, Liu X, Shi Y, Zhang C, Kong L and Li R: The potential application of strategic released apigenin from polymeric carrier in pulmonary fibrosis. Exp Lung Res 43: 359-369, 2017.

28. Bauer D, Redmon N, Mazzio E and Soliman KF: Apigenin inhibits $\mathrm{TNF} \alpha / \mathrm{IL}-1 \alpha$-induced CCL2 release through IKBK-epsilon signaling in MDA-MB-231 human breast cancer cells. PLoS One 12: e0175558, 2017.
29. Ai XY, Qin Y, Liu HJ, Cui ZH, Li M, Yang JH, Zhong WL, Liu YR, Chen S, Sun T, et al: Apigenin inhibits colonic inflammation and tumorigenesis by suppressing STAT3-NF- $\mathrm{KB}$ signaling. Oncotarget 8: 100216-100226, 2017.

30. Han Y, Zhang T, Su J, Zhao Y, Chenchen, Wang and Li X Apigenin attenuates oxidative stress and neuronal apoptosis in early brain injury following subarachnoid hemorrhage. J Clin Neurosci 40: 157-162, 2017

31. Zhang T, Su J, Guo B, Wang K, Li X and Liang G: Apigenin protects blood-brain barrier and ameliorates early brain injury by inhibiting TLR4-mediated inflammatory pathway in subarachnoid hemorrhage rats. Int Immunopharmacol 28: 79-87, 2015.

32. Hernandez ME, Martinez-Mota L, Salinas C, Marquez-Velasco R, Hernandez-Chan NG, Morales-Montor J, Pérez-Tapia M, Streber ML, Granados-Camacho I, Becerril E, et al: Chronic stress induces structural alterations in splenic lymphoid tissue that are associated with changes in corticosterone levels in wistar-kyoto rats. Biomed Res Int 2013: 868742, 2013.

33. Pang Q, Zhang H, Chen Z, Wu Y, Bai M, Liu Y, Zhao Y, Tu F, Liu C and Chen X: Role of caveolin-1/vascular endothelial growth factor pathway in basic fibroblast growth factor-induced angiogenesis and neurogenesis after treadmill training following focal cerebral ischemia in rats. Brain Res 1663: 9-19, 2017.

34. Zhang Q, Wang X, Bai X, Xie Y, Zhang T, Bo S and Chen X: Resveratrol reversed chronic restraint stress-induced impaired cognitive function in rats. Mol Med Rep 16: 2095-2100, 2017.

35. Gassen NC, Hartmann J, Zschocke J, Stepan J, Hafner K, Zellner A, Kirmeier T, Kollmannsberger L, Wagner KV, Dedic N, et al: Association of FKBP51 with priming of autophagy pathways and mediation of antidepressant treatment response: Evidence in cells, mice, and humans. PLoS Med 11: e1001755, 2014.

36. Alcocer-Gomez E, Casas-Barquero N, Williams MR, RomeroGuillena SL, Cañadas-Lozano D, Bullón P, Sánchez-Alcazar JA Navarro-Pando JM and Cordero MD: Antidepressants induce autophagy dependent-NLRP3-inflammasome inhibition in Major depressive disorder. Pharmacol Res 121: 114-121, 2017.

37. Li Q, Liu Y and Sun M: Autophagy and Alzheimer's disease. Cell Mol Neurobiol 37: 377-388, 2017.

38. Karabiyik C, Lee MJ and Rubinsztein DC: Autophagy impairment in Parkinson's disease. Essays Biochem 61: 711-720, 2017.

39. Jia J and Le W: Molecular network of neuronal autophagy in the pathophysiology and treatment of depression. Neurosci Bull 31: 427-434, 2015

40. Huang R and Liu W: Identifying an essential role of nuclear LC3 for autophagy. Autophagy 11: 852-853, 2015.

41. Dunlop EA and Tee AR: mTOR and autophagy: A dynamic relationship governed by nutrients and energy. Semin Cell Dev Biol 36: 121-129, 2014

42. Kim J, Kundu M, Viollet B and Guan KL: AMPK and mTOR regulate autophagy through direct phosphorylation of Ulk1. Nat Cell Biol 13: 132-141, 2011.

43. Rabanal-Ruiz Y, Otten EG and Korolchuk VI: mTORC1 as the main gateway to autophagy. Essays Biochem 61: 565-584, 2017.

44. Xu S, Li L, Li M, Zhang M, Ju M, Chen X and Gu H: Impact on autophagy and Ultraviolet B induced responses of treatment with the MTOR inhibitors rapamycin, everolimus, Torin 1, and pp242 in human keratinocytes. Oxid Med Cell Longev 2017: 5930639, 2017.

45. Hau AM, Greenwood JA, Löhr CV, Serrill JD, Proteau PJ, Ganley IG, McPhail KL and Ishmael JE: Coibamide A induces mTOR-independent autophagy and cell death in human glioblastoma cells. PLoS One 8: e65250, 2013. 